\title{
Stenoz Oluşmuş Y-Şeklinde Bir Damarın Akışkan-Katı Etkileşiminin OpenFOAM ile Analizi
}

\author{
Murad Kucur $^{1 *}$ \\ 1* İstanbul Üniversitesi-Cerrahpaşa Üniversitesi, Mühendislik Fakültesi, Makine Mühendisliği Bölümü, İstanbul, Türkiye, (ORCID: 0000-0002-0356-0359), \\ kucur@iuc.edu.tr
}

(International Conference on Design, Research and Development (RDCONF) 2021 - 15-18 December 2021)

(DOI: $10.31590 /$ josat.1040121)

ATIF/REFERENCE: Kucur, M. (2021). Stenoz Oluşmuş Y-Şeklinde Bir Damarın Akışkan-Katı Etkileşiminin OpenFOAM ile Analizi. Avrupa Bilim ve Teknoloji Dergisi, (32), 871-877.

$\ddot{O} z$

Bu çalışmada Y-şeklinde katı damar modeli açık kaynak kodlu Salome program ile oluşturulmuş, stenöz gözlenen bir dalda akışkankatı etkileşim analizi yapılmıştır. Hesaplamalı akışkanlar dinamiği analizleri OpenFOAM (foam-extend) kullanılarak gerçekleştirilmiştir. Akışkan için hem Newtonyen hem de Newtonyen olmayan akış modelleri kullanılmıştır. Hız girişi ise literatürden pulsatil bir çevrim olarak alınmıştır. Analiz sonucunda stenoz oluşmuş bölgede duvar kayma gerilmeleri ve duvar deformasyonu tespit edilmeye çalışılarak, stenoz olan bölgenin akış üzerindeki etkisi açıklanmıştır.

Anahtar Kelimeler: Stenoz, Damar, OpenFOAM

\section{Fluid-Solid Interaction of a Stenosed Y-Shaped Vessel with OpenFOAM}

\begin{abstract}
In this study, a Y-shaped solid vessel model was created with the open-source Salome program, and fluid-structure interaction analysis was performed in a branch with stenosis. Computational fluid dynamics analyzes were performed by using OpenFOAM (foam-extend). Both Newtonian and Non-Newtonian flow models are used for the fluid. The velocity input is taken as a pulsatile cycle from the literature. As a result of the analysis, the wall shear stresses and wall deformation in the stenosed region were tried to be determined, and the effect of the stenosed region on the flow was explained.
\end{abstract}

Keywords: Stenose, Vessel, OpenFOAM

\footnotetext{
*Sorumlu Yazar: kucur@iuc.edu.tr
} 


\section{Giriş}

Damar içerisinde yă̆ birikmesi sonucu oluşan plaklanma, damar içerisinde stenoz olarak da bilinen arter darlığı yaratır. $\mathrm{Bu}$ durumda ciddi hastalık yaratan bir sorun olabilir. Aterosklerotik plak oluşumları ile çatallanma ve arterlerdeki kan akışı arasındaki ilişki birçok araştırmacı tarafından teorik olarak açıklanmıştır (Ku, 1997; Caro vd., 1971; Malek vd.,1999).

Stenoz oluşan arterin kan akışına etkisini inceleyen araştırmalar, duvar kayma gerilmelerinin (WSS) darlığın derecesine göre değiştiğini göstermiştir (Bit ve Chattopadhyay, 2014; Bit vd., 2017; Bit vd., 2020; Bit ve Chattopadhyay, 2018). Ateroskleroz, damar sertliği ile de ilişkili (Oberoi vd, 2013; Palombo ve Kozakova, 2016) olduğundan damar duvarlarını rijit kabul etmekten ziyade akışkan-katı etkileşim analizleri gerçekleştirmek daha doğru sonuçlar elde edilmesine olanak sağlayacaktır.

Damar duvarının esnekliğini araştıran akışkan-katı etkileşimi (FSI) çalışmalarında damar duvarı için farklı elastisite modülleri dikkate alınarak damar duvarlarındaki deformasyon bulunmuştur. Lopes vd. (2019) yalnızca akışkan davranışına dayalı tipik bir hesaplamalı akışkanlar dinamiği (HAD) problemi ile, akışkan ve katı etkileşiminin dikkate alındığı bir FSI problemi arasında karşılaştırmalı bir çalışma sunmuştur. Rijit ve elastik duvar çalışmaları karşılaştırıldığında, rijit yaklaşımda duvar kayma gerilmeleri (WSS) profillerinin önemli ölçüde daha yüksek tahmin edildiğini belirtmişler ve daha yüksek elastisite modülünün damar duvarlarında daha düşük yerdeğiştirmeler ve daha yüksek akış hızı ürettiği sonucuna varmışlardır. Zaman ortalamalı duvar kayma gerilmesinin (TAWSS) daha düşük değerlerinin karotis sinüsün plak biriktirmeye ve ateroskleroz geliştirmeye daha yatkın bir yer olduğunu gösterdiğini belirtmişlerdir. Kan akışı aslında tam olarak Newtonyen bir davranış göstermez. Literatürdeki çalışmalarda, kan hücrelerinin damar çapına göre küçük olduğu $(\mathrm{Ku}, 1997)$ ve damarların uzun olduğu durumlarda kan akışının Newtonyen kabul edilebileceği söylenmiştir. Kan akışının Newtonyen olmayan davranışını modellemek üzere yapılan araştırmalarda farklı modeller kullanılmıştır. Lopes vd. (2020) farklı viskozite modellerinin etkisini göz önünde bulundurarak karotis arterde FSI çalışması gerçekleştirmişlerdir. Sabit dinamik viskoziteli Newtonyen model ile Carreau-Yasuda Newtonyen olmayan akış modelini karşılaştırmışlardır. Çalışmalarının sonuçlarına göre, Carreau modelinin duvar kayma gerilmesi sonuçları, Newtonyen modele göre kardiyak döngü sırasında biraz daha yüksek çıkmıştır. Ancak, damar duvarının yerdeğiştirmesi viskozite modellerinden etkilenmemiştir. Wong vd. (2013), daha önce Tada ve Tarbell (2005) tarafindan kullanılan bir geometri ile FSI çalışması yapmışlardır. Kan basıncı, stenoz sonucu daralma ve deformasyon arasında bir ilişki kurmaya çalışmışlardır. Sonuçları ile stenoz olan bölgede maksimum duvar kayma gerilmeleri oluştuğunu ve plak oluşumundan sorumlu olabileceğini göstermişlerdir.

Literatürde hastaya özel bilgisayarlı tomografi verilerinden oluşturulan üç boyutlu damar modelleri ile yapılan FSI çalışmaları da bulunmaktadır. Kumar vd. (2020), bilgisayarlı tomografi verilerinden 2-Boyutlu tarama görüntülerinin MIMICS kullanılarak 3-Boyutlu bir modele dönüştürülmesiyle oluşturulan bir hasta karotis arteri kullanarak 3-Boyutlu bir FSI çalışması gerçekleştirmişlerdir.
$\mathrm{Bu}$ çalışmada $\mathrm{Y}$ şeklinde tasarlanmış ve dallarından biri üzerinde stenoz oluşmuş bir damarın katı modeli oluşturularak, açık kaynak kodlu hesaplamalı akışkanlar dinamiği yazılımı olan OpenFOAM ile analizi gerçekleştirilmiştir.

\section{Materyal ve Metot}

\subsection{Modelleme}

Şekil 1, incelenen ana damarın ve dallarının geometrisini ve Şekil 2 ise damarın ağ oluşturulmuş yapısını göstermektedir. Salome ile oluşturulan 3-Boyutlu katı model geometrisinde her üç dalda dairesel olarak modellenmiştir. Ana damarın dış ve iç çapı sırasıyla $8.98 \mathrm{~mm}$ ve $8.33 \mathrm{~mm}$ dir. Stenoz oluşmuş arterin dış ve iç çapları sırasıyla $6,73 \mathrm{~mm}$ ve $5,97 \mathrm{~mm}$, diğer dalın dış ve iç çapları sırasıyla $6,81 \mathrm{~mm}$ ve $6 \mathrm{~mm}$ 'dir. İki dal arasındaki çatallanma açısı 40 derece olarak alınmıştır. Stenozlu bölgede \%70 oranında daralma mevcuttur. Stenoz uzunluğu damar yarıçapının iki katı olarak alınmıştır(Zhang vd,2016).

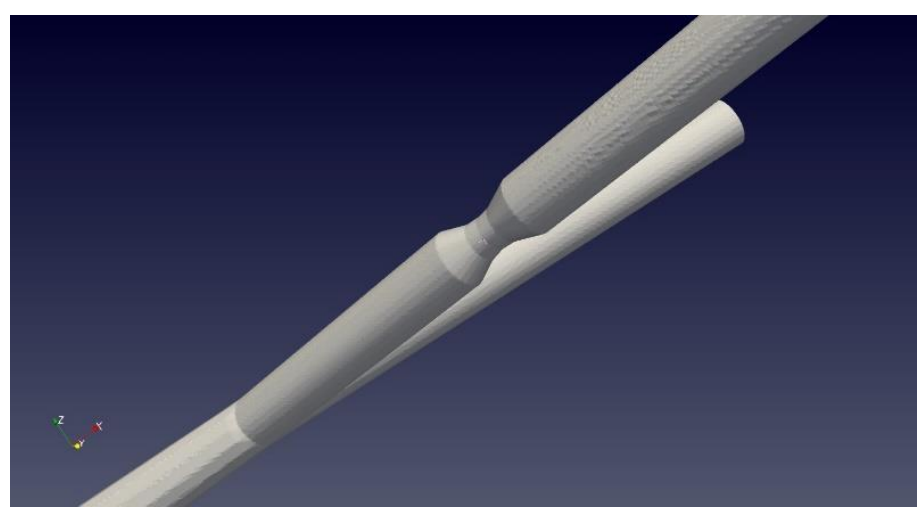

Şekil 1. Damarın katı model görüntüsü

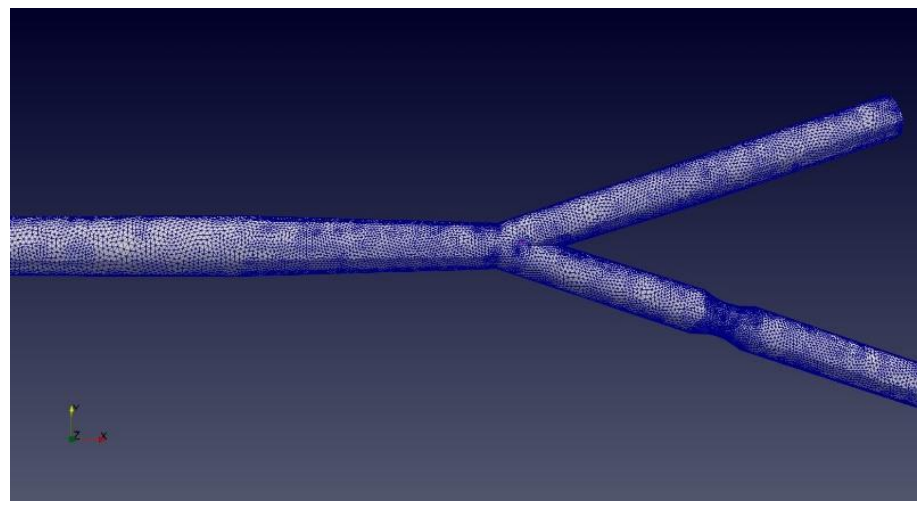

Şekil 2. Damarın ağ oluşturulmuş görüntüsü

Çözümün doğruluğunu belirlemek için çatallanma noktasındaki ortalama yer değiştirme için 0,2 ila 0,95 milyon arasında değişen tetrahedral eleman kullanılarak ağdan bağımsızlık çalışması yapılmıştır. Şekil 3' de gösterilen ağdan bağımsızlık çalışmasının sonuçlarına göre damar duvarı için 250192 eleman ve akışkan alan için 822504 eleman ile oluşturulan ağ yapısının yeterli olduğuna karar verilmiştir. 


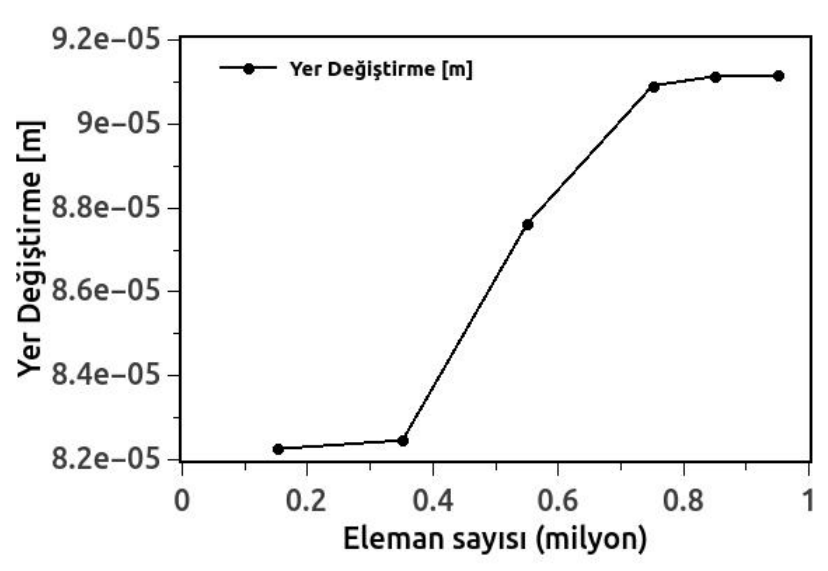

Şekil 3. Ağdan bağımsızlık çalışması

\subsection{Temel Denklemler ve Sınır Koşulları}

Bu çalışmada damar içerisinde kan akışının sıkıştırılamaz ve laminer olduğu varsayılmıştır. Akışkan için hem Newtonyen hem de Newtonyen olmayan akış modelleri ayrı ayrı incelenmiştir. Newtonyen olmayan durum için Carreau modeli kullanılmıştır. $\mathrm{Bu}$ model, hemodinamik simülasyonlarda yaygın olarak kullanılmaktadır.

Damar lineer izotropik malzeme olarak kabul edilerek, damarın mekanik özellikleri $\rho_{w}=1160 \mathrm{~kg} / \mathrm{m}^{3}, v=0.45$ dır. Burada $\rho_{w}$, damar duvarının yoğunluğu ve $v$, Poisson oranıdır. Elastisite modülü, rijitliğin duvar kayma gerilmelerine etkisini araştırmak için $\mathrm{E}=1.106 \times 10^{6} \mathrm{~Pa}$, ve $\mathrm{E}=1.106 \times 10^{6}$ Pa'nın \%25 ve \%75'i gibi farklı değerler olarak alınmıştır. Kanın yoğunluğu ve dinamik vizkozitesi sirasıyla $\rho=1060 \mathrm{~kg} / \mathrm{m}^{3}$ ve $\mu=3.71 \times 10^{-3} \mathrm{~kg} /$ $m$ sn dir.

Newtonyen olmayan durum için, Carreau Modeli

$$
\frac{\eta-\eta_{\infty}}{\eta_{0}-\eta_{\infty}}=\left(1+(k \dot{\gamma})^{a}\right)^{n-1 / a}
$$

dir. Burada k bir gevşeme zaman sabiti (s), n güç indeksi, $\dot{\gamma}$ kesme hızıdır, $\eta_{0}$ kesme hızının sıfır olduğu yerde limit viskozite, $\eta_{\infty}$ kesme hızının sonsuza gittiği yerde limit viskozitesidir. Kan akışının reolojik akış parametreleri $\mathrm{a}=2, \eta=0.056 \mathrm{~Pa} . \mathrm{s}$, $\eta_{\infty}=0.00345 \mathrm{~Pa}$. s, k=3.13 s, n=0.22'dir. Newtonyen akışkanlar için $n=1$ dir. $0<n<1$ ise akışkan pseudoplastiktir ve $1<\mathrm{n}<\infty$ ise kayma altında kalınlaşan akışkandır. Kan akışı için $n<1$ (Cho ve Kensey, 1991)

Akış alanı için yönetici denklemler süreklilik ve momentum denklemleridir. Süreklilik denklemi

$$
\nabla \cdot \mathbf{u}=0
$$

Momentum denklemi,

$$
\rho\left(\frac{\partial \mathbf{u}}{\partial \mathrm{t}}+\mathbf{u} \cdot \nabla \mathbf{u}\right)=-\nabla \mathbf{P}+\mu \nabla^{2} \mathbf{u}+\mathbf{f}
$$

Burada $\mathrm{u}$, hız vektörü, $\mathrm{P}$ basınç ve f gravitasyonel kuvvettir. Damar duvarındaki deformasyon için ise yönetici denklem lineer momentum denklemidir (Lopes vd., 2019),

$$
\rho_{w} \frac{\partial^{2} w}{\partial t^{2}}-\nabla \sigma=\rho_{w} \vec{b}
$$

şeklinde ifade edilir. Burada $w$, arter duvarındaki yerdeğiştirme, $\vec{b}$ katı üzerindeki cisim kuvvetleri, $\sigma$ ise Cauchy gerilme tensörüdür. Lineer elastik izotropik katı için gerilme tensörü (Lopes vd., 2019),

$$
\sigma=2 \mu_{L} \varepsilon+\lambda_{L} \operatorname{tr}(\varepsilon) I
$$

dir. $\mu_{L}$ ve $\lambda_{L}$ sirasiyla birinci ve ikinci Lame parametreleri, $\varepsilon$ gerinme tensörü, tr iz fonksiyonu ve $I$ ise birim matristir. Lame parametreleri elastisite modülü ve Poisson oranının fonksiyonu olarak

$$
\begin{aligned}
& \lambda_{L}=\frac{v E}{(1+v)(2 v-1)} \\
& \mu_{L}=\frac{E}{2(1+v)}
\end{aligned}
$$

şeklindedir. Duvar kayma gerilmesi, kanın arterlerin yüzeyine uyguladığı birim alan başına sürtünme kuvvetidir. Bu çalışmada duvar kayma gerilmeleri

$$
\overrightarrow{\tau_{w}}=\mu \cdot\left(\omega_{\text {wall }} \cdot \vec{n}\right)
$$

denkleminden hesaplanmıştır. Burada $\omega_{\text {wall }}$, duvardaki vortisitedir, $\vec{n}$ ise arter duvarının normal birim vektörüdür.

Akışkan-katı etkileşim problemlerinde ne Lagrange ne de Euler formülasyonu problem alanını çözmek için tam olarak uygundur. Lagrange formülasyonunda, izlenen noktalar ağ alanına sabitlenirken, Euler formülasyonunda noktalar sabit bir ağ alanı boyunca hareket eder. Bu nedenle, Lagrange formülasyonu katılar için kullanılırken, Euler formülasyonu akışkan için kullanılır. Bununla birlikte, akışkan-katı etkileşim problemlerinde, hareketli sınırları olan bir akışkan modeli için Euler formülasyonu, probleminin çözümünü karşılayamaz. Bu durumda, Arbitrary Lagrangian-Eulerian (ALE) yöntemi olarak bilinen hibrit bir formülasyon, FSI problemlerinin yönetim denklemlerini çözmek için daha kullanışlıdır. Burada akışkan alanın, yapısal alanların deformasyonuna göre keyfi olarak deforme olmasına izin verilir. ALE yöntemi ile akışkan ve damar duvarı deformasyonunun temel denklemlerini çözmek yeterli değildir. Akışkan-katı etkileşim bölgesinin matematiksel olarak tanımlanması da gereklidir. Bu çalışmada, akışkan-katı etkileşim bölgesi, OpenFOAM'da foam extend modülü içerisine yerleştirilen IQN-LS (Interface Quasi Newton Method using Least Squares) yöntemi ile çözülmüştür. Ayrıca geometri üzerindeki elastik duvar varsayımı nedeniyle, katının herhangi bir yönde deforme olmasına izin veren serbest deformasyon koşulu uygulanmıştır. Akışkan üzerindeki yerdeğiştirme ve kuvvetler, etkileşim bölgesinde katı üzerindeki yerdeğiştirme ve kuvvetlerine eşit alınmıştır.

Genel olarak, basit kan akışı modelleri daimî akışı dikkate alır. Bununla birlikte, gerçekte kardiyak çevrimin periyodik yapısı, daimî olmayan bir pulsatil akışa neden olur ve pulsatil akışın, akış hızları ve gerilme dağılımları üzerinde önemli etkileri vardır. Bu çalışmada damar girişinde sınır koşulu olarak pulsatil hız girişi alınmıştır ve Şekil 4 de gösterilmiştir. Zaman adımı 0.01 
sn'dir. Burada periyot, her bir periyodik döngü için antrenman koşulunda dakikada 120 kalp atış hızına karşılık gelen 0.5 saniye olarak alınmıştır. Pulsatil akış için maksimum hız $0.5 \mathrm{~m} / \mathrm{sn}$ ve minimum hiz $0,1 \mathrm{~m} / \mathrm{sn}$ 'dir.

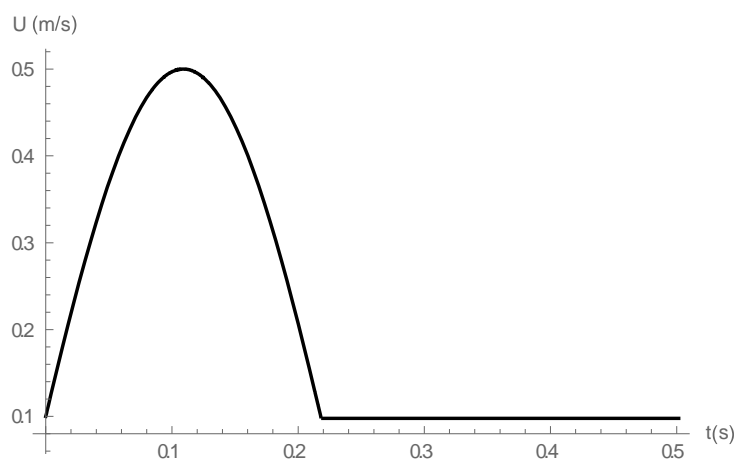

Şekil 4. Pulsatil hız girişi

Pulsatil kan akışı için denklem literatürden (Nagargoje ve Gupta, 2020; Sinnot,2006) Denklem (9)'da gösterildiği gibi alınmıştır:

$U=$

$\left\{\begin{array}{rr}0.5 \sin [4 \pi(t+0.0160236)] & 0.5 n \leq t \leq 0.5 n+0.218 \\ 0.1 & 0.5 n+0.218<t \leq 0.5(n+1)\end{array}\right.$

\section{Araștırma Sonuçları ve Tartıșma}

$\mathrm{Bu}$ çalışmada gerçekleştirilen akışkan-katı etkileşim analizi ile stenoz olmuş bölgedeki duvar kayma gerilmeleri ve çatallanma bölgesinde de yer değiştirmeler elde edilmiştir.

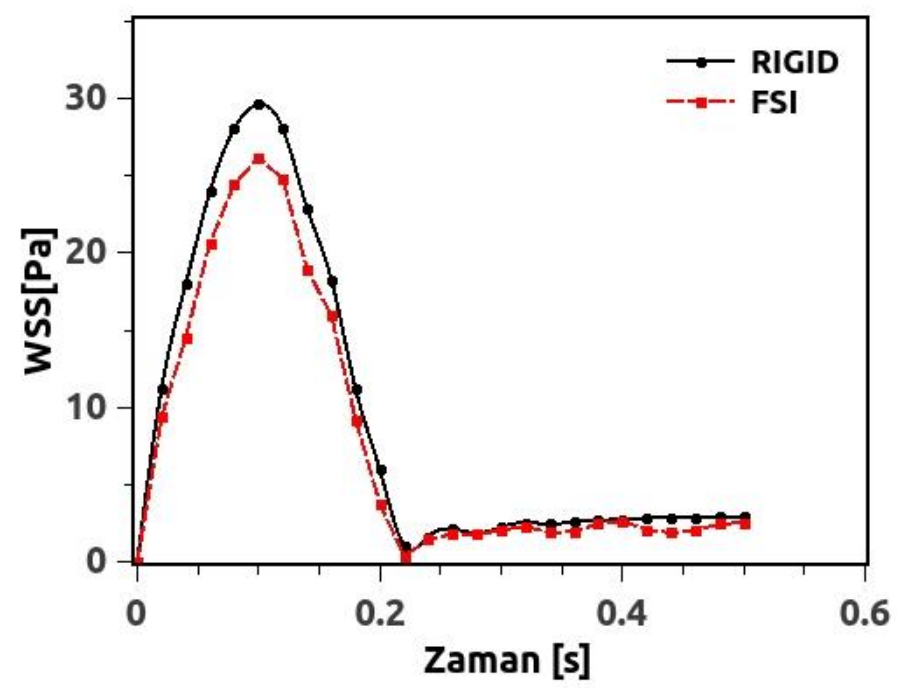

Şekil 5. Rijit ve akışkan-katı etkileşim analizi modellerinden elde edilen duvar kayma gerilmeleri

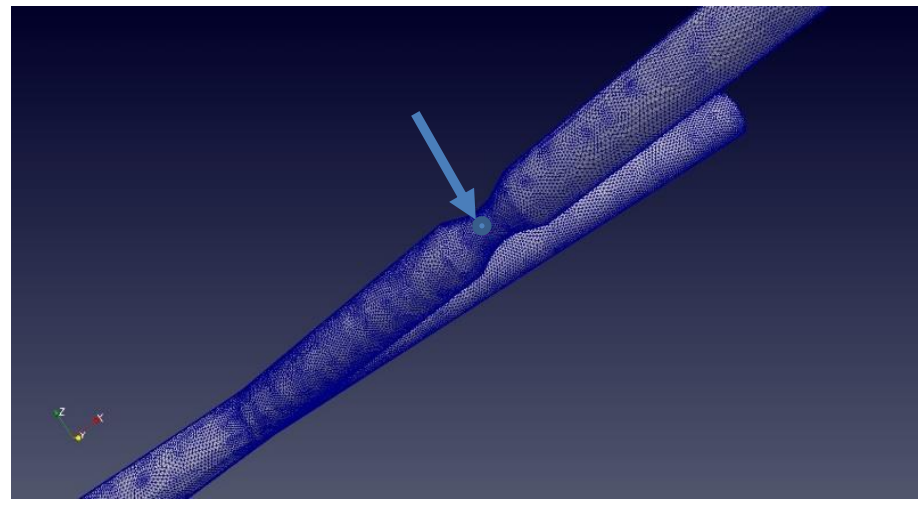

Şekil 6. Rijit ve akışkan-katı etkileşim analizi modellerinden elde edilen duvar kayma gerilme değerlerinin alındığı nokta

Şekil 5’ de stenoz bölgesinde dış duvar çeperinde oluşan kayma gerilmelerinin hem akışkan-katı etkileşimi (FSI) hem de rijit durum için karşılaştırılması gösterilmiştir. Şekil 6' da kayma gerilmelerinin alındığı nokta gösterilmiştir. Duvar kayma gerilmesi değişimi, kan akışının pulsatil döngüsüne oldukça benzer bir şekle sahiptir. Buradaki rijit model, literatürde [Lopes vd., 2019; Wong, 2013; Zhao vd., 2020; Mortazavinia, 2012) bahsedilen önceki araştırmalarda olduğu gibi, FSI yaklaşımından daha yüksek duvar kayma gerilmeleri sonucu vermiştir. Duvar kayma gerilmeleri, sistolün zirvesine yakın pulsatil döngü sırasında en yüksek değerlerini alır. Sistol zirvesinden sonra duvar kayma gerilmeleri azalma eğilimi gösterir.

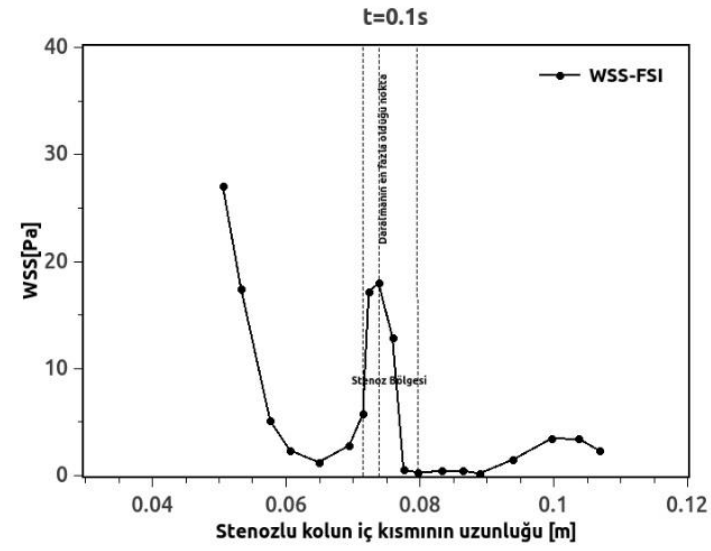

Şekil 7. İç duvar boyunca $\mathrm{t}=0.1 \mathrm{~s}$ deki wss değişimi

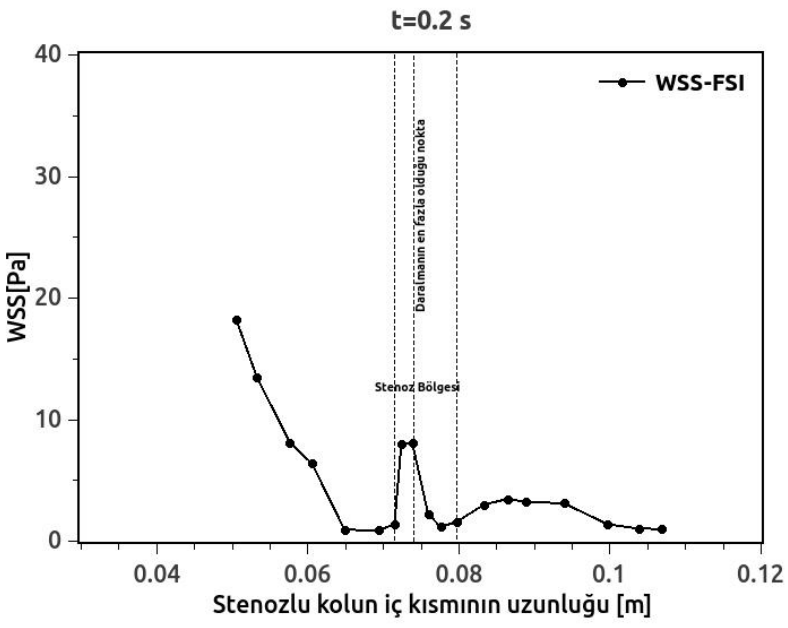

Şekil 8. İç duvar boyunca $\mathrm{t}=0.2 \mathrm{~s}$ deki wss değişimi 


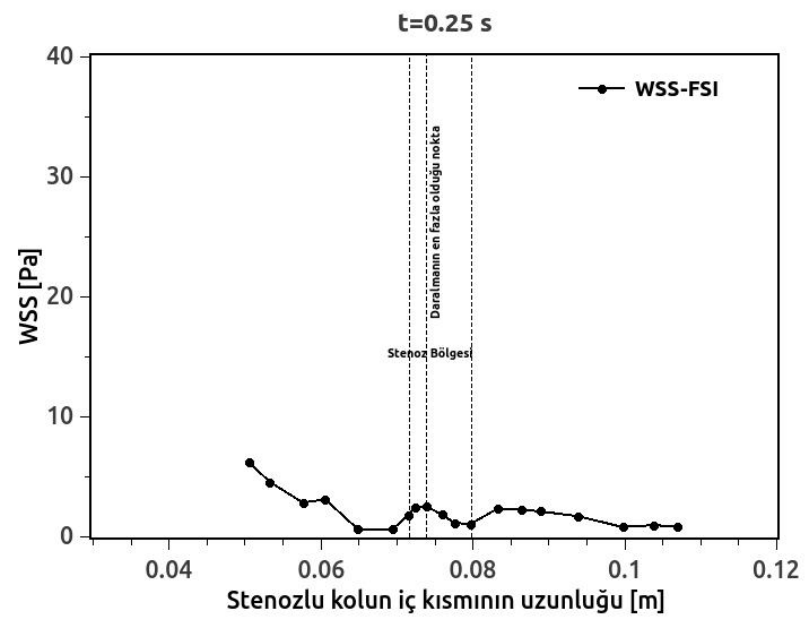

Şekil 9. İç duvar boyunca $\mathrm{t}=0.25 \mathrm{~s}$ deki wss değişimi

Şekil 7-8-9-10 'da WSS değerinin stenozlu damar kolunun iç kısmında konuma göre değişimi $\mathrm{t}=0,1 \mathrm{~s} \mathrm{t}=0,2 \mathrm{~s}, \mathrm{t}=0.25 \mathrm{~s}$ ve $\mathrm{t}=0,3$ s zamanlarında gösterilmiştir. Duvar kayma gerilmeleri stenoz bölgesinde daha yüksek olmaktadır.Sistol anında diğer zamanlara göre en yüksek wss ler elde edilirken, diastol anında ise wss değerlerinde azalma görülmektedir.

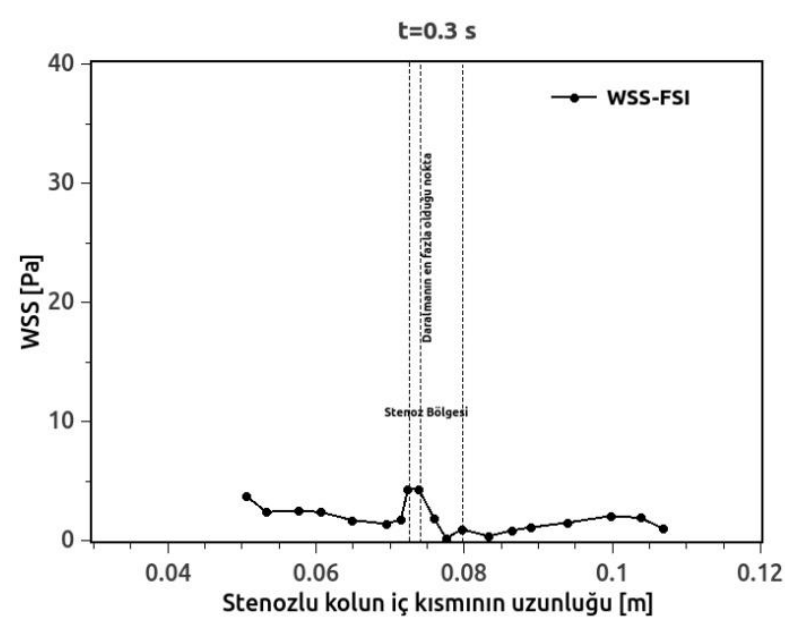

Şekil 10. İç duvar boyunca $t=0.3 \mathrm{~s}$ deki wss değişimi

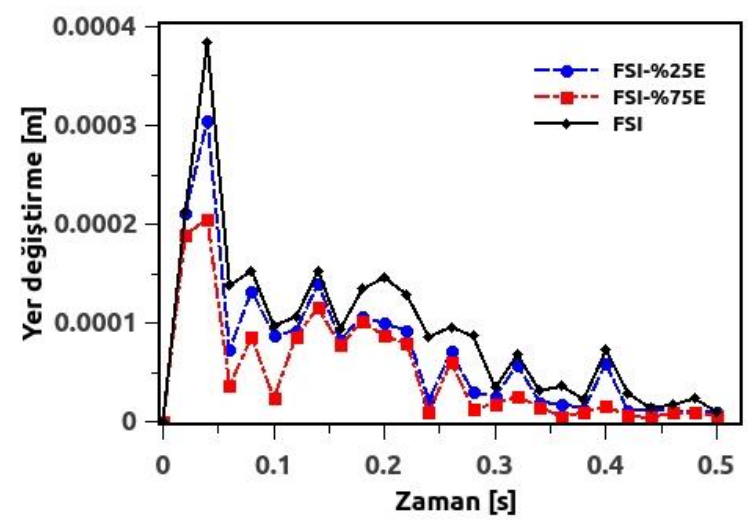

Şekil 11. Farklı elastisite modülleri için yerdeğiştirmelerin analizi

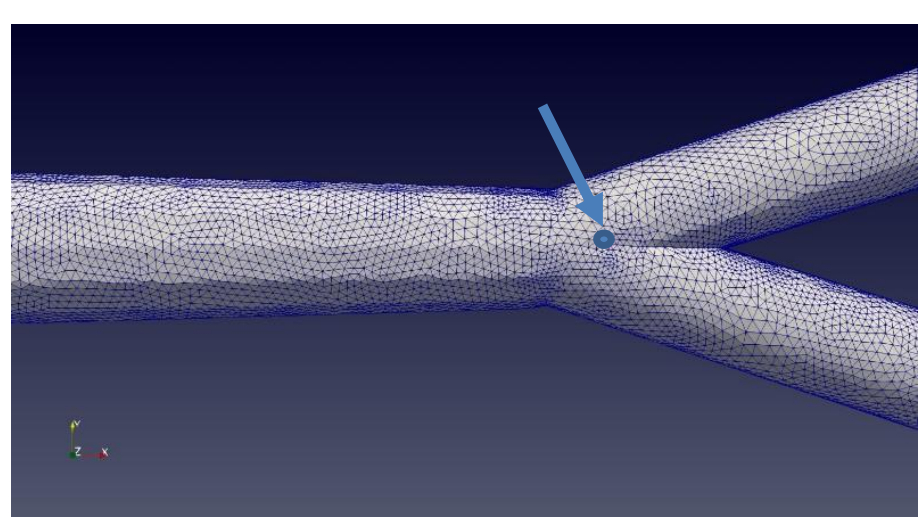

Şekil 12. Yer değiştirme değerlerinin alındığı nokta

Şekil 11' de, E $=1.106 \times 106 \mathrm{~Pa}, \% 25 \mathrm{E}$ ve $\% 75 \mathrm{E}$ gibi farklı elastisite modülü değerleri için yerdeğiştirmeleri göstermektedir. Şekil 12' de yer değiştirmelerin alındığı çatallanma bölgesindeki nokta gösterilmektedir. Yaş ilerledikçe damarlar elastikiyetini kaybetmektedir. Damar duvarlarının elastikiyetinin yerdeğiştirme ve duvar kayma gerilmeleri üzerindeki etkisi önem kazanmaktadır. Beklendiği gibi, bu çalışmada da elde edilen sonuçlarda, elastisite modülü arttıkça damar duvarlarının yerdeğiştirme miktarı azalmaktadır.

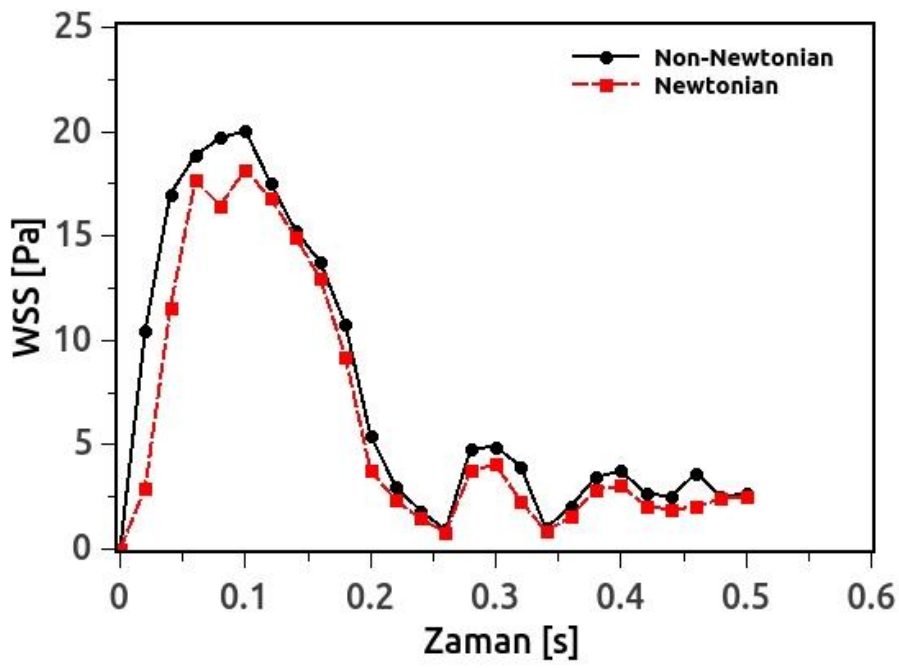

Şekil 13. Newtonyen ve Newtonyen olmayan akış modellerinden elde edilen duvar kayma gerilmeleri

Şekil 13, hem Newtonyen hem de Newtonyen olmayan akış için akışkan-katı etkileşim modelinden elde edilen duvar kayma gerilmelerini göstermektedir. Newtonyen olmayan akış durumunda, duvar kayma gerilmeleri Newtonyen durumdan daha yüksektir.

\section{Sonuç}

Düşük duvar kayma gerilimi alanları damarlarda plak oluşumundan sorumludur. Çatallanma bölgeleri ile stenöz olmuş alanlar plak oluşumuna uygun noktalardır. Çünkü bu bölgelerdeki akış ayrılması ve pulsatil döngü sırasında damarın dış duvarındaki duvar kayma gerilmelerinin iç duvardaki duvar kayma gerilmelerinden daha düşük olması bu duruma neden olur.

$\mathrm{Bu}$ çalışmada, açık kaynaklı bir tasarım programı olan Salome ile 3-Boyutlu Y-şeklinde bir damar geometrisi 
oluşturulmuştur. Daha sonra, akışkan-katı etkileşimi dikkate alınarak, pulsatil döngü sırasında duvar kayma gerilmelerinin ve duvar yer değiştirmelerinin zamana bağlı değişimi incelenmiştir. İlerleyen yaşa bağlı olarak damar rijitliğinde meydana gelen artışı gözlemlemek için damar duvarı farklı elastisite modüllerinde alınmış ve farklı elastisite modülünün duvar yerdeğiştirmeleri üzerindeki etkileri ortaya konmuştur. Literatürdeki benzer bir modele atıfta bulunarak, ana damar girişinde pulsatil bir hız girişi sınır koşulu olarak kullanılmıştır (Nagargoje ve Gupta, 2020; Sinnot, 2006). Hesaplamalı akışkanlar mekaniği analizleri açık kaynak kodlu OpenFOAM programı kullanılarak gerçekleştirilmiştir.

$\mathrm{Bu}$ araştırmada, pulsatil döngü boyunca yerdeğiştirme ve duvar kayma gerilmelerinin zamana göre değişimi, kan akışının pulsatil döngüsüne benzer bir formada meydana gelmiştir. Rijit model, FSI modelinden daha yüksek duvar kayma gerilmeleri öngörmüştür. Elastisite modülünü arttırmak damar duvarlarının yerdeğiştirmesini azaltmıştır. Newtonyen olmayan akış durumu için elde edilen duvar kayma gerilmeleri, Newtonyen akış modeli ile elde edilen değerlerin biraz üzerindedir. $\mathrm{Bu}$ çalışmada elde edilen sonuçlar literatürdeki temel bilgilerle uyumludur.

\section{Kaynakça}

1. Ku, D.N. (1997). Blood flow in arteries. Annu Rev of Fluid Mech, 29, 399-434.

2. Caro, C.G., FitzGerald, J.M., ve Schroter, R.C. (1971). Atheroma and arterial wall shear observation, correlation and proposal of a shear dependent mass transfer mechanism for arterogenesis. P Roy Soc Lond B Bio, 1046(177), 109-133.

3. Malek, A.M., Alper, S.I., ve Izumo, S. (1999). Hemodynamic shear stress and its role in atherosclerosis. The Journal of the American Medical Association JAMA, 282(21), 2035-2042.

4. Bit, A. ve Chattopadhyay, H. (2014). Numerical investigations of pulsatile flow in stenosed artery. Acta Bioeng Biomech, 16(4), 33-44.

5. Bit, A., Ghagare, D., Rizvanov, A.A., ve Chattopadhyay, H. (2017). Assessment of influences of stenoses in right carotid artery on left carotid artery using wall stress marker. Bio Med Research International, 2017, 1-13.

6. Bit, A., Alblawi, A., Chattopadhyay, H., Quais, Q.A., Benim, A.C., Rahimi-Gorji, M., ve Do, H.T. (2020). Threedimensional numerical analysis of hemodynamic of stenosed artery considering realistic outlet boundary conditions. Comput Meth Prog Bio, 185, 105163.

7. Bit, A. ve Chattopadhay, H. (2018). Acute aneurysm is more critical than acute stenoses in blood vessels: a numerical investigation using stress markers. Bio Nano Sci, 8, 329-336.

8. Oberoi, S., Schoepf, U.J., Meyer, M., Henzler, T., Rowe, G.W., ve Costello, P. (2013). Progression of arterial stiffness and coronary atherosclerosis: Longitudinal evaluation by cardiac cT. Am J.Roentgenol, 200(4), 798-804.

9. Palombo, C. ve Kozakova, M. (2016). Arterial stiffness, atherosclerosis and cardiovascular risk: Pathophysiologic mechanisms and emerging clinical indications. Vascul Pharmacol, 77, 1-7.

10. Lopes, D., Puga, H., Teixeira, J.C., ve Teixeria, S.F. (2019). Influence of arterial mechanical properties on carotid blood flow: comparison of CFD and FSI studies. International Journal of Mechanical Sciences, 160, 209-218.

11. Lopes, D., Puga, H., Teixeira, J.C., ve Teixeria, S.F. (2020). Fluid-structure interaction study of carotid blood flow: comparison between viscosity models. Eur $J$ of Mech BFluid, 83, 226-234.

12. Wong, K.K.L., Thavornpattanapong, P., Cheung, S.C.P., ve Tu, J.Y. (2013). Biomechanical investigation of pulsatile flow in a three-dimensional atherosclerotic carotid bifurcation model. J Mech in Med Biol, 13, 1-21.

13. Tada, S. ve Tarbell, M. (2005). A computational study flow in a compliant carotid bifurcation-stress phase angle correlation with shear stress. Annals of Biomedical Engineering, 33(9), 1202-1212.

14. Kumar, N., Khader, A.S.M, Pai, R., Khan, S.H., ve Kyriacou, P.A. (2020). Fluid structure interaction study of stenosed carotid artery considering the effects of blood pressure. International Journal of Engineering Science, 154, 1-14.

15. Zhang Q., Zhang Y., Zhou Y., Zhang K., Zhang Ke. ve Gao L.(2016). An ultrasounsd simulation model for the pulsatile blood flow modulated by the motion of stenosed vessel wall. BioMed Research International, 2016,1-16.

16. Cho, Y. ve Kensey, K.R. (1991). Effects of the non-newtonian viscosity of blood on flows in a diseased arterial vessel Part 1: Steady flows. Biorheology, 28, 241-262.

17. Nagargoje, M. ve Gupta, R. (2020). Effect of sinus size and position on hemodynamics during pulsatile flow in a carotid artery bifurcation. Comput Meth Prog Bio, 192, 105440.

18. Sinnot, M., Cleary, P.W., ve Prakash, M. (2006). An investigation of pulsatile blood flow in a bifurcation artery using a grid-free method. Fifth International Conference on CFD in the Process Industries, Melbourne, Australia.

19. Zhao, S.Z., Xu, X.Y., Hughes, A.D., Thom, S.A., Stanton, A.V., Ariff, B., ve Long, Q. (2000). Blood flow and vessel mechanics in a physiologically realistic model of a human carotid arterial bifurcation. J Biomech, 33, 975-984.

20. Mortazavinia, Z., Goshtasbi, E.R., Emdad, H., Sharifkazemi, M.B., Zare, A., ve Mehdizadeh, A.R. (2012). Study of pulsatile non-newtonian blood flow through abdominal aorta and renal incorporating fluid-structure interaction. $J$ Biomed Phys Eng., 2, 93-102. 